Article

\title{
Vacuum Interaction of Crossed Cosmic Strings
}

\author{
Irina G. Pirozhenko ${ }^{1,2}$ (D)
}

check for

updates

Citation: Pirozhenko, I.G. Vacuum Interaction of Crossed Cosmic Strings. Universe 2021, 7, 217. https:/ / doi.org/ 10.3390 /universe7070217

Academic Editor: Galina L.

Klimchitskaya

Received: 28 May 2021

Accepted: 25 June 2021

Published: 30 June 2021

Publisher's Note: MDPI stays neutral with regard to jurisdictional claims in published maps and institutional affiliations.
1 Bogoliubov Laboratory of Theoretical Physics, Joint Institute for Nuclear Research, Joliot-Curie 6, 141980 Dubna, Russia; pirozhen@theor.jinr.ru

2 Department of Fundamental Problems of Microworld Physics, Dubna State University, Universitetskaya 19, 141982 Dubna, Russia

\begin{abstract}
In this paper, we consider the vacuum energy of a scalar field in the spacetime of two non-parallel cosmic strings. To this end, we obtain metrics for orthogonal straight cosmic strings and for slightly nonparallel strings. In the first case, we derive the separation-dependent part of the vacuum energy in the leading order of string tension. The dependence of the vacuum energy on separation differs from that known for parallel strings. For two strings inclined at a small angle to each other, the approximation used simply reproduces the result for parallel strings, since the angle dependence enters the next to leading order. The results are compared with the Casimir interaction between two inclined cylinders.
\end{abstract}

Keywords: vacuum energy; cosmic string; conical singularity; renormalization

\section{Introduction}

Symmetry-breaking phase transitions in the early universe may cause the formation of cosmic strings which are linear defects, characterized by a dimensionless parameter $G \lambda / c^{2}=\Lambda^{2} / M_{P l}^{2}$, where $\lambda$ is the mass per unit length of strings (equal to the string tension), $G$ is the Newton constant, $M_{p l}$ is the Planck mass, and $\Lambda$ is the energy scale of symmetry-breaking. First models of cosmic strings considered symmetry-breaking at the grand unification scale with estimated string tension $G \lambda / c^{2} \sim 10^{-6}$. It was supposed that such strings could give origin to structure formation. Later, they were ruled out by the acoustic peaks in the CMB power spectrum. Analysis of the CMB anisotropy data obtained by the Planck collaboration gives a limitation on the effective mass density of a string, which for different models does not exceed $10^{-7}[1,2]$.

Line sources in General Relativity were discussed in [3]. It was noticed that topologically stable strings do not have ends. They can either form closed loops or extend to infinity. The energy momentum tensor for a straight infinite string parallel to $z$ axis is given by

$$
T_{\mu v}(x, y)=\lambda c^{2} \delta(x) \delta(y) \times \operatorname{diag}(1,0,0,-1),
$$

where $\lambda$ is the linear energy density. The metric of the spacetime with one cosmic string may be found in linear approximation of general relativity, $g_{\mu v}=\eta_{\mu v}+h_{\mu v}$, with $\eta_{\mu v}=\operatorname{diag}(1,-1,-1,-1)$ and $h_{\mu v}<<1$, where in harmonic gauge, $\partial_{\mu} h_{\nu}^{\mu}-\frac{1}{2} \partial_{\nu} h_{\mu}^{\mu}=0$, the Einstein equations read [4]

$$
\square h_{\mu v}=-16 \pi \frac{G}{c^{2}}\left(T_{\mu v}-\frac{1}{2} \eta_{\mu v} T\right) .
$$

The solutions corresponding to the energy momentum tensor (1) are

$$
h_{00}=h_{33}=0, \quad h_{11}=h_{22}=8 G \lambda \ln (r / \rho),
$$

where $r=\sqrt{x^{2}+y^{2}}$, and $\rho$ is an arbitrary parameter, which implies finite string thickness. This metric describes geometry at distances larger than $\rho$. For cosmological strings born 
because of the spontaneous symmetry-breaking in GUT, one can estimate $\rho \sim 10^{-29} \mathrm{~cm}$, $\lambda \sim 10^{22} \mathrm{~g} / \mathrm{cm}$. A spacetime of an infinitely thin cosmic string is a conical space with the angle deficit $\delta \varphi=8 \pi G \lambda / c^{2}$. The metric is locally flat, but has a conical singularity at $r=0$.

In cosmology, cosmic strings are often viewed not individually, but as an ideal gas. The energy-momentum tensor of an ideal gas of infinite cosmic strings is obtained by applying the Lorentz boost to the initial string configuration parallel to the $z$ axis. After averaging over the directions of the boost, we arrive at the equation of state for the gas of cosmic strings [5].

The static scalar Newton potential of a cosmic string $U$, defined by $g_{00}=1+2 U$, vanishes. Therefore, there is no gravitational interaction of a neutral classical particle with a straight cosmic string. The same goes for two parallel straight cosmic strings. (This is not valid for curved strings). However, there may exist observable effects, such as lensing, distortion of $\mathrm{CMB}$, and extra density of matter in the wake of a moving cosmic string.

To date, no evidence of cosmic strings has been observed. Despite this, the search for cosmic strings and other topological defects is included in the physics program of leading international collaborations. The main directions are gravitational lensing, analysis of the $\mathrm{CMB}$ anisotropy, and detection of gravitational waves generated by cosmic strings.

The current upper bound on the energy scale of the possible cosmic string network from pulsar timing observations is $G \lambda / c^{2} \sim 10^{-11}$. Unfortunately, at this level and below, no effects can be seen in the $\mathrm{CMB}$, and the strings cannot be discovered by gravitational lensing (except certain microlensing models). Other possible gravitational effects of strings are also significantly limited by this bound. New limits on cosmic strings from gravitational wave observation were set in 2018. [2].

Nevertheless, the classical cosmic string background affects the fluctuations of quantum fields. In one-loop approximation, the contributions of scalar massless, neutrino, and electromagnetic fields to the vacuum polarisation in the gravitational field of a cosmic string were found in [6]. The UV-divergences of the effective action for fields with different spins on manifolds with conical singularities were studied in [7] by making use of the heat kernel expansion.

Cosmic strings may interact with particles and each other due to fluctuations of quantum fields. The vacuum energy of the scalar field in the background of two parallel cosmic strings was first derived by Bordag in 1990 [8]. The dependence of this energy on the string separation results in attractive Casimir force between the strings. This problem was studied by different methods in [9-11]. We also mention the Casimir-Polder force acting on a polarizable particle in the geometry of a straight cosmic string [12].

Non-parallel moving cosmic strings were considered primarily in connection with the so-called Gott time-machine. In [13], Gott demonstrated that pairs of moving cosmic strings may produce closed timelike curves. He proposed to obtain solutions for moving and crossed strings from a static solution for equal parallel strings at $x= \pm x_{0}$, by applying to $x \leq 0$ and $x \geq 0$ half-spaces Lorentz boosts, rotations, and translations that map the flat hypersurface $x=0$ into itself.

In this article, we consider the vacuum interaction of crossed static cosmic strings. In Section 2, we first discuss the metric of a static spacetime with many parallel cosmic strings. Then we move on to non-parallel but non-intersecting strings. In Section 3, we give a derivation of the vacuum energy for a scalar field in spacetime with two non-parallel cosmic strings. In conclusion, we discuss whether it is possible to use the proximity force approximation for two strings close to parallel, and summarize.

Throughout this paper, we use the units $G=\hbar=c=1$.

\section{Spacetime of Multiple Infinite Cosmic Strings}

The spacetime of $\mathrm{N}$ strings parallel to the $z$ axis is defined as a solution of the Einstein equations with the energy-momentum tensor 


$$
T_{\mu v}(x, y)=\sum_{i=1}^{N} \lambda_{i} \delta\left(x-x_{i}\right) \delta\left(y-y_{i}\right) \times \operatorname{diag}(1,0,0,-1) .
$$

Parallel cosmic strings can be effectively reduced to zero-dimensional objects and point-like masses. The corresponding metric in $D=2+1$ was obtained using the SchwarzChistoffel formula [14].

For $N$ parallel straight cosmic strings, the metric is given by [15]

$$
d s^{2}=d t^{2}-d z^{2}-e^{-4 \sum_{i} V_{i}}\left(d x^{2}+d y^{2}\right),
$$

with

$$
V_{i}=2 \lambda_{i} \ln r_{i}, \quad r_{i}=\left[\left(x-x_{i}\right)^{2}+\left(y-y_{i}\right)^{2}\right]^{1 / 2} .
$$

Here, $\lambda_{i}$ is a mass per unit length of a string, $\left(x_{i}, y_{i}\right)$ are the coordinates of the $i$-th string on the $(\mathrm{x}, \mathrm{y})$ plane. The spacetime is locally flat, except for $N$ conical singularities, each with angle deficit $8 \pi \lambda_{i}$. For an open spacetime, the total angle deficit is less than $2 \pi$, so $\sum_{i=1}^{N} \lambda_{i}<1 / 4$.

Obviously, in the case of cosmic stings tilted with respect to each other, the problem cannot be reduced to a lower-dimensional one. If the angle between two non-intersecting strings is $\pi / 2$, then one needs to look for the metric as a solution to the Einstein equations with the energy-momentum tensor of the form

$$
\begin{aligned}
T_{\mu v}(x, y) & =\lambda_{1} \delta\left(x-x_{1}\right) \delta\left(y-y_{1}\right) \times \operatorname{diag}(1,0,0,-1) \\
& +\lambda_{2} \delta\left(x-x_{2}\right) \delta\left(z-z_{2}\right) \times \operatorname{diag}(1,0,-1,0) .
\end{aligned}
$$

This energy-momentum tensor corresponds to geometry. When two strings lie in two parallel planes, the first string is parallel to the $z$ axis, and the second one is parallel to the $y$ axis. Solving the linearized Einstein Equation (2) with the energy-momentum tensor (5) in harmonic gauge, one finds the perturbation $h_{\mu v}$ above the Minkowski metric $\eta_{\mu v}$. For two perpendicular straight static cosmic strings, it reads

$$
h_{00}=0, \quad h_{11}=h_{22}+h_{33}, \quad h_{22}=8 \lambda_{1} \ln \left(r_{1} / \rho_{0}\right), \quad h_{33}=8 \lambda_{2} \ln \left(\tilde{r}_{2} / \rho_{0}\right),
$$

where $\rho_{0}$ is a constant standing for the string radius, $r_{1}$ and $\tilde{r}_{2}$ denote the positions in $(x, y)$ and $(z, x)$ planes, respectively,

$$
r_{1}=\left[\left(x-x_{1}\right)^{2}+\left(y-y_{1}\right)^{2}\right]^{1 / 2}, \quad \tilde{r}_{2}=\left[\left(z-z_{2}\right)^{2}+\left(x-x_{2}\right)^{2}\right]^{1 / 2}, \quad x_{1} \neq x_{2} .
$$

We used tilded letters to distinguish between perpendicular planes.

Using the same reasoning as [4], we can find that for two perpendicular disjoint infinitely thin strings, the spacetime metric is determined by the expression

$$
d s^{2}=d t^{2}-e^{-4\left(V_{1}(x, y)+\tilde{V}_{2}(x, z)\right)} d x^{2}-e^{-4 V_{1}(x, y)} d y^{2}-e^{-4 \tilde{V}_{2}(x, z)} d z^{2},
$$

where

$$
V_{1}=2 \lambda_{1} \ln r_{1}, \quad \tilde{V}_{2}=2 \lambda_{2} \ln \tilde{r}_{2},
$$

and the determinant of the metric is $g=-e^{-8\left(V_{1}+\tilde{V}_{2}\right)}$. This metric has two conical singularities with angle deficits in planes $(x, y)$ and $(x, z)$. Setting $\lambda_{2}=0$, we obtain the metric of a single string parallel to the $z$ axis, and vice versa, for $\lambda_{1}=0$, we restore the metric of a single string parallel to the $y$ axis.

In the case of an arbitrary angle between the strings, the problem of finding the metric becomes trickier. In 1993, Gal'tsov and Letelier [16] generalized the approach of [14] and demonstrated how to obtain the spacetime of multiple moving crossed cosmic strings from the Minkowski spacetime

$$
d s^{2}=d t^{2}-d z^{2}-d Z d \bar{Z}
$$


by singular coordinate transformation (Christoffel-Schwarz formula)

$$
Z(\zeta)=X+i Y=\int_{\zeta_{0}}^{\zeta} \prod_{i=1}^{N}\left(\xi-\alpha_{i}(t, z)\right)^{-4 \lambda_{i}} d \xi, \quad \zeta=x+i y
$$

This transformation takes into account that in general, the position of the $j$-th string depends on time and the third space coordinate $z$,

$$
\alpha_{j}=a_{j}+i b_{j}=v_{x, j} t+w_{x, j} z+x_{0, j}+i\left(v_{y, j} t+w_{y, j} z+y_{0, j}\right) .
$$

Here we use the notations of [16], and $v_{x, j}$ and $v_{y, j}$ denote the velocities of the strings, and $w_{x, j}$ and $w_{y, j}$ are the inclinations of the strings with respect to the $z$ axis.

The line element is

$$
d s^{2}=d t^{2}-d z^{2}-e^{-4 V}(d \zeta+F d t+G d z)(d \bar{\zeta}+\bar{F} d t+\bar{G} d z),
$$

where $V=\sum_{i=1}^{N} \lambda_{i} \ln \left|\zeta-\alpha_{i}\right|^{2}$, and the functions $F$ and $G$ are defined by the integrals:

$$
F=\prod_{i=1}^{N}\left(\zeta-\alpha_{i}\right)^{4 \lambda_{i}} \int_{\zeta_{0}}^{\zeta} \frac{d \xi}{\prod_{k=1}^{N}\left(\xi-\alpha_{k}\right)^{4 \lambda_{k}}} \sum_{j=1}^{N} \frac{4 \lambda_{j} \dot{\alpha}_{j}}{\zeta-\alpha_{j}}, \quad G=\left.F\right|_{\dot{\alpha}_{j} \rightarrow \dot{\alpha}_{j}} .
$$

Here, $\zeta_{0}$ is some constant, and we assume $\left|\zeta_{0}\right| \rightarrow \infty$. One can show that $F$ and $G$ are regular functions of $\zeta[16]$,

$$
F\left(\zeta=\alpha_{i}\right)=-\dot{\alpha}_{i}, \quad G\left(\zeta=\alpha_{i}\right)=-\dot{\alpha}_{i},
$$

and their derivatives up to any order can be computed at $\zeta=\alpha_{i}$.

The case of two parallel moving strings was analyzed in [16]. See also the discussion in [17]. Here, we focus on two nonparallel static strings. We do not consider the case when strings intersect.

The position of two static strings, $v_{x, j}=v_{y, j}=0, j=1,2$, is given by

$$
\alpha_{j}=w_{x, j} z+x_{0, j}+i\left(w_{y, j} z+y_{0, j}\right)
$$

therefore $\dot{\alpha}=0, \dot{\alpha} \neq 0, F=0, G=G_{1}+i G_{2}$, and the line element is

$$
d s^{2}=d t^{2}-d z^{2}\left(1+G_{1}^{2}+G_{2}^{2}\right)-e^{-4 V}\left\{d x^{2}+d y^{2}+2 G_{1} d x d z+2 G_{2} d y d z\right\} .
$$

Let $\alpha_{1}=-\alpha_{2}=\alpha=a+i w z$, where $w=\tan (\theta / 2)$, and $\theta$ stands for the angle between the strings. If $\theta=0$, then $\alpha=a$, the function $G(\zeta)$ vanishes, and one reobtains the metric of parallel strings (3).

For nonparallel equal strings with $\lambda_{1}=\lambda_{2}=\lambda$,

$$
G(\alpha, \zeta)=8 \lambda \alpha \alpha^{\prime}\left(\zeta^{2}-\alpha^{2}\right)^{4 \lambda} \int_{\zeta_{0}}^{\zeta} \frac{d \xi}{\left(\xi^{2}-\alpha^{2}\right)^{4 \lambda+1}}
$$

The integration yields the hypergeometric function ${ }_{2} F_{1}(\alpha, \zeta)$. Computing $G(\alpha, \zeta)$ to the first order of $|\alpha / \zeta|$ and in the weak coupling regime, we obtain

$$
G_{1}=8 \lambda m(m z x-a y) / r^{2}, \quad G_{2}=-8 \lambda m(m z y+a x) / r^{2} .
$$

For small-angle $\theta$, the line element is

$$
d s^{2}=d t^{2}-d z^{2}-e^{-4 V}\left\{d x^{2}+d y^{2}-16 \tan (\theta / 2) \lambda a \frac{(y d x+x d y)}{r^{2}} d z\right\},
$$


where $2 a$ is the closest separation of the strings, $V=\lambda \ln \left(r_{1} r_{2}\right)$, and $r_{1}=\ln \left[(x-a)^{2}+y^{2}\right]$, $r_{2}=\ln \left[(x+a)^{2}+y^{2}\right]$. With the substitution

$$
d z \rightarrow d z-8 e^{-4 \lambda V} \tan (\theta / 2) \lambda a \frac{(y d x+x d y)}{r^{2}},
$$

the metric can be rewritten as

$$
d s^{2}=d t^{2}-d z^{2}-e^{-4 V}\left\{d x^{2}+d y^{2}-(8 \lambda \delta)^{2} e^{-4 V} \frac{(y d x+x d y)^{2}}{r^{4}}\right\}
$$

and we introduced the notation

$$
\delta=\tan (\theta / 2) a
$$

The determinant of the metric is

$$
g=-e^{-8 V}\left(1-(8 \lambda \delta)^{2} e^{-4 V} / r^{2}\right) .
$$

\section{Vacuum Interaction of Two Cosmic Strings}

There is no classical gravitational interaction between straight cosmic strings, and they do not radiate gravitational energy; however, they interact due to quantum vacuum fluctuations.

We consider the fluctuations of a scalar field in the background of two nonparallel cosmic strings. The action of a scalar field is given by

$$
S=-\frac{1}{2} \int d^{4} x \sqrt{-g} \phi(x) \Delta \phi(x),
$$

where $\Delta$ is the Laplace-Beltrami operator,

$$
\Delta=\nabla_{\mu} \nabla^{\mu}=\frac{1}{\sqrt{-g}} \partial_{\mu} \sqrt{-g} g^{\mu v} \partial_{\nu}
$$
tor $(26)$

We compute the vacuum energy in terms of the functional determinant of the opera-

$$
\mathcal{E}_{0}=-\frac{i}{2 T} \ln \operatorname{det} \Delta, \quad T=\int d t .
$$

To this end, we decompose the operator $\mathcal{K}$ as

$$
\Delta=\mathcal{K}+\delta \mathcal{K},
$$

where $\mathcal{K} \equiv \partial^{2}=\partial_{t}^{2}-\partial_{x}^{2}-\partial_{y}^{2}-\partial_{z}^{2}$ is the flat spacetime $\mathrm{d}^{\prime}$ Alembertian, and $\delta \mathcal{K}$ is the perturbation due to the strings.

For two parallel strings, this perturbation in the leading order of $\lambda_{1}$ and $\lambda_{2}$ is [10]

$$
\delta K_{\|}=\left(e^{-4\left(V_{1}+V_{2}\right)}-1\right)\left(\partial_{1}^{2}+\partial_{2}^{2}\right)=-4\left(V_{1}+V_{2}\right)\left(\partial_{x}^{2}+\partial_{y}^{2}\right)+\mathcal{O}\left(\lambda^{2}\right) .
$$

If the strings are perpendicular, then using the metric (8), we obtain

$$
\delta K_{\perp}=-4\left[V_{1}(x, y)\left(\partial_{x}^{2}+\partial_{y}^{2}\right)+\tilde{V}_{2}(x, z)\left(\partial_{x}^{2}+\partial_{z}^{2}\right)\right]+\ldots
$$

For two equal strings with a small angle $\theta$ between them, the perturbation $\delta K$ derived by making use of the metric (22) reads 


$$
\begin{aligned}
\delta K & =\delta K_{\|}+\delta K_{\theta} \\
\delta K_{\theta} & =-\frac{16 \delta^{2}}{r^{4}} \lambda^{2}\left[\left(y \partial_{x}+x \partial_{y}\right)^{2}+\frac{2}{r^{2}}\left(x\left(x^{2}-3 y^{2}\right) \partial_{x}+y\left(y^{2}-3 x^{2}\right) \partial_{y}\right)\right]+\mathcal{O}\left(\lambda^{3}\right)
\end{aligned}
$$

with $\delta$ given by (23). Here, we keep the second order of the expansion, because it is in this order that the angular dependence appears.

Following [10], we expand ln det with respect to the perturbation $\delta \mathcal{K}$

$$
\begin{aligned}
\ln \operatorname{det} \Delta & =\ln \operatorname{det}\left[\partial^{2}+\delta \mathcal{K}\right]=\ln \operatorname{det} \partial^{2}+\ln \operatorname{det}\left[1+\partial^{2} \delta \mathcal{K}\right] \\
& =-\operatorname{tr} \ln \partial^{-2}+\operatorname{tr} \partial^{-2} \delta \mathcal{K}-\frac{1}{2} \operatorname{tr} \partial^{-2} \delta \mathcal{K} \partial^{-2} \delta \mathcal{K} .
\end{aligned}
$$

The first and second terms in (32) correspond to tadpole graphs which are put to zero for a massless field in dimensional regularization. In the second order of the perturbation theory, we are left with the third term of (32).

\subsection{Parallel Strings}

In the case of parallel strings, $\delta \mathcal{K}$ is given by (29). Then, in the lowest order of the parameters $\lambda_{1}$ and $\lambda_{2}$, the distance-dependent part of the vacuum energy is

$$
\mathcal{E}_{||}=\frac{i}{2 T} \operatorname{tr}\left(\partial^{-2} \delta \mathcal{K}_{1} \partial^{-2} \delta \mathcal{K}_{2}\right), \quad \delta \mathcal{K}_{a}=-4 V_{a}\left(\partial_{x}^{2}+\partial_{y}^{2}\right) .
$$

Here, the terms $\partial^{-2} \delta \mathcal{K}_{1} \partial^{-2} \delta \mathcal{K}_{1}$ and $\partial^{-2} \delta \mathcal{K}_{2} \partial^{-2} \delta \mathcal{K}_{2}$ are omitted, as they do not depend on the distance between strings and do not contribute to the force.

In [10], the distance-dependent part of the vacuum energy given by (33) was derived in dimensional regularization,

$$
\mathcal{E}_{\|}^{r e n}=-\frac{4}{15 \pi} \frac{\lambda_{1} \lambda_{2}}{(2 a)^{2}} \int d z
$$

This result coincides with the Casimir energy obtained as a vacuum expectation value of the energy momentum tensor derived in $[9,11]$. The distance dependence changes with the spacetime dimension. The vacuum energy for two cosmic strings in the spacetime of arbitrary dimension was derived in [9].

\subsection{Perpendicular Strings}

Let us compute the distance-dependent part of the vacuum energy for perpendicular cosmic strings using the approach of the paper [10]. In this case, the perturbation $\delta K$ is given by (30) and the distance-dependent part of the vacuum energy reads

$$
\mathcal{E}_{\perp}=\frac{i}{2 T} \operatorname{tr}\left(\partial^{-2} \delta \mathcal{K}_{1} \partial^{-2} \delta \mathcal{K}_{2}\right),
$$

where

$$
\delta \mathcal{K}_{1}=-4 V_{1}(x, y)\left(\partial_{x}^{2}+\partial_{y}^{2}\right), \quad \delta \mathcal{K}_{2}=-4 \tilde{V}_{2}(x, z)\left(\partial_{x}^{2}+\partial_{z}^{2}\right) .
$$

By the trace, we mean $\operatorname{tr} \mathcal{M}=\int d^{4} x \sqrt{-g} \mathcal{M}$, and in the given order of $\lambda_{i}$ we assume $\sqrt{-g} \sim 1$. The trace is computed in momentum representation

$$
\mathcal{E}_{\perp}=\frac{8 i}{T} \int \frac{d^{4} k}{(2 \pi)^{4}} \frac{d^{4} q}{(2 \pi)^{4}} \frac{\left(k_{1}^{2}+k_{2}^{2}\right)}{k^{2}} \frac{\left((q+k)_{1}^{2}+(q+k)_{3}^{2}\right)}{(q+k)^{2}} V_{1}(q) \tilde{V}_{2}(-q) .
$$

Let the first string coincide with the $z$ axis, and the second string lies in the $(x, y)$ plane being parallel to the $y$ axis, 


$$
x_{1}=y_{1}=0, \quad x_{2}=2 a, \quad z_{2}=0 .
$$

Here, $2 a$ is the closest separation between the strings. Then, the Fourier transforms of the functions $V_{1}$ and $\tilde{V}_{2}$, given by (9), are

$$
V_{1}(q)=(2 \pi)^{2} \frac{\lambda_{1}}{q^{2}} \delta\left(q_{0}\right) \delta\left(q_{3}\right), \quad \tilde{V}_{2}(q)=(2 \pi)^{2} \frac{\lambda_{2}}{q^{2}} e^{-2 a i q_{1}} \delta\left(q_{0}\right) \delta\left(q_{2}\right)
$$

Substituting (38) into (39), one arrives at

$$
\mathcal{E}_{\perp}=\frac{8 i \lambda_{1} \lambda_{2}}{(2 \pi)} \int \frac{d q_{1}}{q_{1}^{4}} e^{2 i a q_{1}} \int \frac{d^{4} k}{(2 \pi)^{4}} \frac{\left(\left(k_{1}-q_{1}\right)^{2}+k_{2}^{2}\right)}{\left(k-q_{\perp}\right)^{2}} \frac{k_{1}^{2}+k_{3}^{2}}{k^{2}},
$$

where $q_{\perp}=\left(0, q_{1}, 0,0\right)$. We compute the integral over $k$ in dimensional regularization, replacing $d^{4} k \rightarrow d^{n} k, n=4-2 \varepsilon$. After the Wick rotation, $k_{0}=i k_{4}, d^{n} k=i d^{n} k_{E}$, the integral reads

$$
\begin{aligned}
I_{E}\left(q_{2}\right) & =i \int \frac{d^{n} k}{(2 \pi)^{n}} \frac{\left(k_{1}^{2}+k_{3}^{2}\right)}{k^{2}} \frac{\left(\left(k_{1}-q_{1}\right)^{2}+k_{3}^{2}\right)}{\left(k-q_{\perp}\right)^{2}} \\
& =\frac{i q_{1}^{n}}{(2 \pi)^{n}} \frac{(-\pi)^{\frac{n}{2}}}{\Gamma(n+2)} \Gamma\left(\frac{n}{2}\right)^{2} \Gamma\left(-\frac{n}{2}\right) \frac{n^{2}}{16}\left(2 n^{3}+3 n^{2}-2 n-2\right)
\end{aligned}
$$

In (40) we performed the momentum integration according to (A1) and (A2) in Appendix A. To integrate over $q_{2}$, the $\alpha$-representation is used, and we obtain

$$
\int d q_{1} q_{1}^{n-4} e^{2 i a q_{1}}=\frac{\sqrt{\pi}}{a^{n-3}} \frac{\Gamma(n / 2-3 / 2)}{\Gamma\left(2-\frac{n}{2}\right)} .
$$

The poles of the gamma functions in (40) and (41) mutually cancel. Finally, removing the regularization, $\varepsilon \rightarrow 0$, we get a result for the separation-dependent part of the vacuum energy, which is free of divergences,

$$
\mathcal{E}_{\perp}^{r e n}=\lim _{\mathcal{E} \rightarrow 0} \mathcal{E}_{\perp}^{\varepsilon}=-\frac{83}{240 \pi^{2}} \frac{\lambda_{1} \lambda_{2}}{2 a}
$$

\subsection{Small Angle between the Strings}

Unfortunately, we cannot use this approach when the angle between strings is small, and the metric is given by (22). As it was already mentioned, the angle dependence enters the next to leading order of the expansion (31). Therefore, the leading contribution to the Casimir energy is given by (34) and the next order is proportional to $\theta^{2}$. For small angles between strings, a more fruitful method is to compute the Casimir energy using the vacuum expectation value of the energy momentum tensor expressed in terms of Green's function of the operator $\mathcal{K}[9,11]$.

For two infinitely thin strings inclined at a small angle $\theta$, one may define a PFA-like approximation. Let us treat opposite infinitesimal string segments as parallel and separated by distance $\rho$, which depends on the third coordinate $z$,

$$
\rho=\sqrt{(2 a)^{2}+z^{2} \tan ^{2} \theta}
$$


where $2 a$ is the closest separation between the strings. Then, assuming the additivity of the segment contributions, the vacuum energy of the scalar field in the background of two strings is obtained through integration:

$$
\mathcal{E}_{\theta}=-\int_{-L / 2}^{L / 2} d z \varepsilon_{\|}(\rho)=\mathcal{E}_{\|}(2 a) f(L / 2 a, \theta),
$$

where $L$ is the length of the string, and $\varepsilon_{\|}(\rho)$ is the linear vacuum energy density for parallel strings (34) with the constant distance $2 a$ replaced by $\rho$. The function $f(L / 2 a, \theta)$ shows the ratio of the Casimir energies evaluated for crossed and parallel cosmic strings

$$
f(L / 2 a, \theta)=\frac{4 a}{L \tan (\theta)} \arctan \left(\frac{L \tan (\theta)}{4 a}\right) \simeq 1-\frac{\theta^{2}}{12} \frac{L}{2 a} .
$$

This expression is obtained for small angles and is not valid for perpendicular strings, where $f$ diverges.

\section{Conclusions}

We have considered the vacuum energy of a scalar field in the spacetime of two non-parallel cosmic strings. To this end, we obtained the metrics for perpendicular straight cosmic strings and for slightly nonparallel strings. In the first case, we derived the separation-dependent part of the vacuum energy in the leading order of $\lambda_{1} \lambda_{2}$. This result is non-perturbative with respect to the angle between the strings. The distance dependence of the vacuum energy (42) differs from that known for parallel strings (34). Up to the numerical factor,

$$
\mathcal{E}_{\perp} / \mathcal{E}_{\|} \sim 2 a / L .
$$

The results obtained may be of interest for cosmological models in which strings are formed as a random network of straight moving segments. A certain role in the evolution of this network should be played by quantum effects, in particular, the mutual attraction and rotation of non-parallel strings due to fluctuations of quantum fields, studied in the present work [18].

The method we used here is identical to the TGTG formula or scattering approach. As demonstrated in [11], the potential of multiple cosmic strings has a form suitable for applying the TGTG formula only in the leading order of expansion in $\lambda_{i}$. The reason is that the contributions of conical defects enter the perturbation (28) not additively, but in a more complex way. When we expand $\delta \mathcal{K}$ with respect to the string tension $\lambda$, in the leading order, the contributions split, $\delta \mathcal{K}=\delta \mathcal{K}_{1}+\delta \mathcal{K}_{2}$, and the expressions (33), (36) become applicable.

This approximation is insufficient for close to parallel strings, since the angle dependence enters the beyond-leading order of (31). In this geometry, the leading order reproduces the result for parallel strings. The exact derivation of the vacuum energy in the background of two cosmic strings, inclined at a small angle to each other, is reserved for future work.

For two non-contiguous bodies, it is possible to estimate the Casimir force using the so-called Proximity Force Approximation (PFA). It is valid if the distance between the bodies is much less than the radius of curvature of their surface.

Two infinite cylinders, the axis of the first cylinder being inclined with respect to the axis of the second at an angle $\theta$, is a system somewhat analogous to crossed strings. Like crossed strings, the cylinders are infinite in only one dimension. The difference is that the radius of the cylinders is finite. The space is flat, there are no conical singularities. In [19], the problem of two crossed cylinders was investigated by methods of scattering theory and proximity force approximation was also derived. It was shown that the distance dependence changes significantly in comparison with the PFA for parallel cylinders. Moreover 
the PFA result for inclined cylinders diverges when the angle between them approaches zero. The ratio of the vacuum energies for perpendicular and parallel cylinders is

$$
E_{\perp}^{c y l} / E_{\|}^{c y l}=\frac{8}{3 L} \sqrt{R l} .
$$

Here, $l=2 a-2 R$ is the surface-to-surface gap, and $2 a$ is the closest distance between the cylinders' axes. Compare with the ratio for cosmic strings (43).

In the problem of crossed infinitely thin strings, there is no parameter with respect to which one could determine large and small distances. In the problem of crossed cylinders, this parameter is the ratio of the shortest distance between the cylinders to their radius $R$. The distance is considered small if $l \ll R$. In the problem of crossed strings, such a parameter could be the finite radius of a string.

Funding: This research received no external funding.

Acknowledgments: The author acknowledge discussions with M.Bordag, A.A. Vladimirov and E. Radionova.

Conflicts of Interest: The author declares no conflict of interest.

\section{Appendix A}

To derive (40) we used the following expressions for euclidean momentum integration

$$
\begin{aligned}
\int d^{n} k \frac{k_{\mu} k_{v} k_{\gamma} k_{\rho}}{k^{2}(q-k)^{2}}= & \frac{i(-\pi)^{\frac{n}{2}}\left(q^{2}\right)^{\frac{n}{2}-2}}{\Gamma(n+2)}\left\{q_{\mu} q_{\nu} q_{\gamma} q_{\rho} \Gamma\left(2-\frac{n}{2}\right) \Gamma\left(\frac{n}{2}+3\right) \Gamma\left(\frac{n}{2}-1\right)\right. \\
& +\frac{q^{2}}{2} \Gamma\left(1-\frac{n}{2}\right) \Gamma\left(\frac{n}{2}+2\right) \Gamma\left(\frac{n}{2}\right) g_{[\mu \nu} q_{\rho} q_{\gamma]} \\
& \left.+\frac{q^{4}}{4} \Gamma\left(\frac{n}{2}+1\right)^{2} \Gamma\left(-\frac{n}{2}\right)\left(g_{\mu \nu} g_{\gamma \rho}+g_{\mu \gamma \gamma} g_{v \rho}+g_{\mu \rho \rho} g_{v \gamma}\right)\right\}, \\
\int d^{n} k \frac{k_{\mu} k_{\nu} k_{\gamma}}{k^{2}(q-k)^{2}}= & \frac{i(-\pi)^{\frac{n}{2}}\left(q^{2}\right)^{\frac{n}{2}-2}}{\Gamma(n+1)}\left\{q_{\mu} q_{\nu} q_{\gamma} \Gamma\left(2-\frac{n}{2}\right) \Gamma\left(2+\frac{n}{2}\right) \Gamma\left(\frac{n}{2}-1\right)\right. \\
& \left.+\frac{q^{2}}{2} \Gamma\left(1-\frac{n}{2}\right) \Gamma\left(1+\frac{n}{2}\right) \Gamma\left(\frac{n}{2}\right)\left(q_{\mu} g_{\nu \lambda}+q_{\nu} g_{\mu \lambda}+q_{\lambda} g_{\mu \nu}\right)\right\} .
\end{aligned}
$$

Here $g_{[\mu v} q_{\rho} q_{\gamma]}$ denotes 6 terms of this kind with all nonidentical index permutations.

\section{References}

1. Ade, P.A.R.; Aghanim, N.; Armitage-Caplan, C.; Arnaud, M.; Ashdown, M.; Atrio-Barandela, F.; Aumont, J.; Baccigalupi, C.; Banday, A.J.; Barreiro, R.B.; et al. Planck 2013 results. XXV. Search for cosmic strings and other topological defects. Astron. Astrophys. 2014, 571, A25.

2. Blanco-Pillado, J.J.; Olum, K.D.; Siemens, X. New limits on cosmic strings from gravitational wave observation. Phys. Lett. B 2018, 778, 392-396. [CrossRef]

3. Israel, W. Line sources in general relativity. Phys. Rev. D 1977, 15, 935-941. [CrossRef]

4. Vilenkin, A. Gravitational field of vacuum domain walls and strings. Phys. Rev. D 1981, 23, 852-857. [CrossRef]

5. Rubakov, V.A.; Gorbunov, D.S. Introduction to the Theory of the Early Universe: Hot Big Bang Theory; World Scientific: Singapore, 2017.

6. Frolov V.P.; Serebryanyi, E.M. Vacuum Polarization in the Gravitational Field of a Cosmic String. Phys. Rev. D 1987, 35, 3779-3782. [CrossRef]

7. Fursaev, D.V.; Miele, G. Cones, spins and heat kernels. Nucl. Phys. B 1997, 484, 697-723. [CrossRef]

8. Bordag, M. On the vacuum interaction of two parallel cosmic strings. Ann. Phys. 1990, 47, 93. [CrossRef]

9. Galtsov, D.V.; Grats, Y.V.; Lavrentev, A.V. Vacuum polarization and topological selfinteraction of a charge in multiconic space. Phys. Atom. Nucl. 1995, 58, 516-521.

10. Grats, Y.V. Vacuum interaction of conic singularities. Teor. Mat. Fiz. 2015, 186, 243-251. [CrossRef]

11. Munoz-Castaneda, J.M.; Bordag, M. Quantum vacuum interaction between two cosmic strings revisited. Phys. Rev. D 2014, 89, 065034. [CrossRef]

12. Saharian, A.A.; Kotanjyan, A.S. Repulsive Casimir-Polder forces from cosmic strings. Eur. Phys. J. C 2011, 71 1765. [CrossRef]

13. Gott, J.R. Closed timelike curves produced by pairs of moving cosmic strings: Exact solutions. Phys. Rev. Lett. 1991, 66 1126-1129. [CrossRef] [PubMed] 
14. Staruszkiewicz, A. Gravitation Theory in Three-Dimensional Space. Acta Phys. Polon. 1963, 24, 735-740.

15. Letelier, P.S. Multiuple Cosmic Strings. Class. Quant. Grav. 1987, 4, L75-L77. [CrossRef]

16. Letelier, P.S.; Galtsov, D.V. Multiple moving crossed cosmic strings. Class. Quant. Grav. 1993, 10, L101-L107. [CrossRef]

17. Khusnutdinov, N. Self-action in gravity. Eur. Phys. J. Plus 2021, 136, 669. [CrossRef]

18. Vilenkin, A.; Levin, Y.; Gruzinov, A. Cosmic strings and primordial black holes. J. Cosmol. Astropart. Phys. 2018, 2018, 8. [CrossRef]

19. Rodriguez-Lopez, P.; Emig, T. Casimir interaction between inclined metallic cylinders. Phys. Rev. A 2012, 85, 032510. [CrossRef] 